\title{
Full-order observer-based output regulation for linear heterogeneous multi-agent systems under switching topology
}

\author{
Yuliang Cai ${ }^{1}$, Qiang $\mathrm{He}^{2, *}$, Jie Duan ${ }^{1}$, Zhiyun $\mathrm{Gao}^{1}$ \\ ${ }^{1}$ College of Information Science and Engineering, Northeastern University, Shenyang, Liaoning, 110004, China \\ Email: ylcaivv@163.com,dj89111@163.com, zhiyungao@163.com \\ ${ }^{2}$ College of Computer Science and Engineering, Northeastern University, Shenyang, 110819, China \\ Email: heqiangcai@gmail.com \\ *Corresponding Author: Qiang He, Email: heqiangcai@ gmail.com
}

How to cite this paper: Cai, Y. L., He, Q., Duan, J., Gao, Z. Y. (2019) Full-order observer-based output regulation for linear heterogeneous multi-agent systems under switching topology. Journal of Artificial Intelligence and Systems, 1, 20-42.

https://doi.org/10.33969/AIS.2019.11002

Received: May 25, 2019

Accepted: June 28, 2019

Published: July 1, 2019

Copyright (c) 2019 by author(s) and Institute of Electronics and Computer. This work is licensed under the Creative Commons Attribution International License (CC BY 4.0)

http://creativecommons.org/licenses/by/4.0/

\section{Open Access}

\begin{abstract}
This study addressed the output regulation issue of linear heterogeneous multi-agent systems under switching topology. All agents excluding the external system are divided into two groups with measurable agents or unmeasurable agents. The agents' states in the first group can be available for measurement while the agents' states in the second group are unmeasurable. For the second group, a full-order Luenberger observer is devised to recover these agents' states. Moreover, there are some agents that can not receive the information from the exosystem directly, thus, a dynamic compensator is constructed for these agents. Based on the proposed observer and compensator, a hybrid feedback control strategy is put forward to settle the output regulation issue. Furthermore, the information interaction among agents is expressed by the switching topology, and the topology is assumed to be jointly connected. Finally, two numerical examples are given to illustrate the feasibility of the theoretical results. The results show that whether the states are measurable or not, the proposed control strategy can address the output regulation issue of linear heterogeneous MASs under switching topology. Moreover, the comparative experiment indicates that our method obtains superior performance in terms of convergence speed, and is more efficient in dealing with practical problems.
\end{abstract}

\section{Keywords}

Full-order observer, Dynamic compensator, Hybrid feedback controller, Switching topology, Output regulation

\section{Introduction}

Over the last few decades, the efficient hierarchical control of industrial plants has achieved remarkable benefits in reducing energy consumption, and improving operational efficiency. More concretely, the industrial plants are generally considered as the large-scale systems with high complexity, large delay, and strong uncertainty. Industrial processes can be divided into several subsystems with different properties, such as energy or information flows [1,2]. When the controlled plant variables increase, the cooperative control of industrial plants 
becomes more and more challenging. In order to solve this problem, many researchers regard industrial subsystems as agents, thus the industrial systems can be considered as multi-agent systems (MASs). The cooperative control of MASs has attracted great attention and has been applied in many fields, such as smart grid, vehicle systems, sensor networks, and mobile robots [3-6].

Due to the uncertainties, complexity, diversity and instability of MASs, output regulation has become an important and challenging direction in cooperative control [7, 8]. Generally speaking, the application objects of the multi-agent distributed output regulation control can be multiple wheeled mobile robots, mass-damper-spring systems, networked aircrafts, and so on [9-11]. These objects can be regarded as agents of MASs, and their structures are usually different with each other. Moreover, in practice, it is difficult to measure all states of objects due to high cost or technical constraint. Furthermore, the information interaction among all objects delivered by the communication topology often changes during the moving of these objects. Therefore, it is an urgent problem to design the distributed control protocol and enable these objects to achieve the output regulation control under above circumstances. Motivated by this, this paper focuses on the distributed output regulation issue of linear heterogeneous MASs with partial unmeasurable agents under switching topology.

Several profound results, which can build the theoretical framework of output regulation control for MASs, have been obtained, see [12-17] and the references therein. Specifically, the output regulation problems for general linear MASs were considered in [12, 13]. As shown in $[12,13]$, the system matrices $A$ and $B$ associated with linear MASs are homogeneous, i.e. the system matrices $A$ and $B$ of all agents are identical. However, such assumption is too restrictive for many real applications. In this case, the output regulation problems of linear heterogeneous MASs were studied in [14-17]. It can be observed that the system dynamics of all agents are heterogeneous, which have addressed the issues of $[12,13]$. Moreover, it should be noted that the system states of all agents in $[12,13]$ play a key role in their control protocols. For this reason, these control strategies are developed on a common assumption that the states of agents can be measured.

In many practical occasions, not all agents' states are measurable due to technical constraint or external disturbance. In this case, several profound results on state observers have been proposed to recover the agents' states [18-23]. For instance, the state observer was put forward for unmeasurable states, and the dynamic output feedback controller based on state observer was designed in [19]. The distributed control law based on adaptive distributed observer was presented for linear MASs [21]. Moreover, a passivity-based distributed observer was designed for each agent to estimate the state information that cannot be available for measurement [23]. From the review of the aforementioned works with state observers, it can be seen that the states of all agents are assumed to be unmeasurable. Nevertheless, in many real-world cases, not all agents are unmeasurable, namely, partial agents are measurable. To our knowledge, there are few reported results on output regulation issue for partial unmeasurable agents, which forms the first motivation of this paper.

Although the output regulation problems for linear MASs with state observers have been investigated [18-23], they are developed on fixed topology. However, in the actual scenario, the communication topologies are often variable. Therefore, the research of output regulation issue under switching topology is more meaningful [24-27]. The works in [24-26] investigated the cooperative output regulation problem of heterogeneous linear MASs under switching topology. However, it is supposed that the states of all agents can be measured. In addition, the system dynamics associated with linear heterogeneous MASs in [26] have the same dimensions. Moreover, the cooperative output regulation issue with state observer under switching network was studied in [27], nevertheless, all agents were assumed to be unmeasurable. To authors' knowledge, the output regulation issue of linear heterogeneous MASs with partial unmeasurable agents under switching topology has not been addressed, which forms the second motivation of this paper.

The main contributions of this note are summarized as follows. Firstly, the partial 
unmeasurable agents are considered in the present work for the first time, while the previous works studied the MASs with all measurable dynamics [12-17] or all unmeasurable dynamics [18-23]. Therefore, the case considered in this study is more general and realistic. Secondly, compared with the previous works [12-23] that were developed on fixed topology, this paper considered the switching topology, which is more flexible and feasible. Thirdly, different from the existing methods, the present work takes the points including heterogeneous MASs with different dimensions, partial unmeasurable agents and switching topology into account at the same time. Therefore, the current result is more general in contrast to the case where all measurable states [12-17], all unmeasurable states [18-23], homogeneous system dynamics [12, 13], heterogeneous system dynamics with the same dimensions [26], or fixed topology [12-23] are considered. Finally, compared with the existing method [17], the proposed method obtains superior performance in terms of convergence speed, and is more efficient in dealing with practical problems.

The rest of this study is organized as follows. Some basic conception of the communication topology and the system dynamics are introduced in Section 2. The hybrid feedback controller is designed in Section 3. The main results are presented in Section 4. In Section 5, two simulation examples are provided to verify the feasibility of the theoretical results. And in Section 6, the conclusion is finally given.

Notations: The symbols $R, R^{n}$ and $R^{n \times m}$ indicate the set of real numbers, $n$-dimension vector space and $n \times m$ matrix space, respectively. $\mathbb{Z}^{+}$and $\mathbb{N}$ indicate the positive integers set and natural numbers set, respectively. For matrix $A, A^{T}$ is the transpose of $A$. The $\mathbf{0}$ indicates the zero matrix with appropriate dimensions. $\mathbf{1}_{N}$ denotes the column vector $(1,1, \ldots, 1)^{T}$. Notations $A \otimes B$ and $A \oplus B$ are the Kronecker product and Kronecker sum of $A$ and $B$, separately. If $A \in R^{n \times n}$ and $B \in R^{m \times m}$, the Kronecker sum $A \oplus B$ is given as $A \oplus B=$ $\left(A \otimes I_{m}\right)+\left(I_{n} \otimes B\right)$. The eigenvalues of $A \oplus B$ are $\lambda_{i}+\mu_{j}, i=1,2, \ldots, n, j=1,2, \ldots, m$, where $\lambda_{i}$ is the eigenvalue of $A$ and $\mu_{j}$ is the eigenvalue of $B$. Moreover, $\sigma(A)$ is used to indicate the spectrum of $A$.

\section{Preliminaries and problem formulation}

\subsection{Algebraic graph theory}

Some basic definitions and results of the communication graph are described in this subsection and the details can be referred to [28].

The communication topology structure can be described by the digraph $\mathscr{G}=\{\mathscr{V}, \mathscr{E}\}$ with a node index set $\mathscr{I}=\{0,1,2, \ldots, N\}$, a vertex set $\mathscr{V}=\left\{v_{0}, v_{1}, \ldots, v_{N}\right\}$ and an edge set $\mathscr{E}=\left\{e_{i j}=\left(v_{i}, v_{j}\right) \in \mathscr{V} \times \mathscr{V}\right\}$. Here, $v_{i}(i=1,2, \ldots, N)$ denotes the $i$-th agent and $v_{0}$ denotes the exosystem. The neighborhood of the $i$-th agent is expressed as $\mathscr{N}_{i}=\left\{v_{j} \in \mathscr{V}\right.$ : $\left.\left(v_{i}, v_{j}\right) \in \mathscr{E}\right\} . \mathscr{A}=\left[a_{i j}\right]$ with $a_{i i}=0(i, j \in \mathscr{I})$ denotes the weighted adjacency matrix. If the edge $e_{i j}=\left(v_{i}, v_{j}\right) \in \mathscr{E}$, then $a_{i j}>0$, else $a_{i j}=0$. Note that the exosystem merely sends information to agents, i.e., $a_{01}=a_{02}=\cdots=a_{0 N}=0$, and if exosystem sends information to agent $i, a_{i 0}>0$, else $a_{i 0}=0$. Therefore, the adjacency matrix can then be defined as

$$
\mathscr{A}=\left(\begin{array}{cc}
0 & \mathbf{0} \\
\mathscr{A}_{0} \mathbf{1}_{N} & \mathscr{A}_{S}
\end{array}\right)
$$

where $\mathscr{A}_{0}=\operatorname{diag}\left\{a_{10}, a_{20}, \ldots, a_{N 0}\right\}$ and $\mathscr{A}_{s}=\left[a_{i j}\right], i, j=1,2 \ldots, N$. Moreover, the in-degree and out-degree of agent $i$ are given

$$
\operatorname{deg}_{\text {in }}\left(v_{i}\right)=\sum_{j=0}^{N} a_{j i}, \operatorname{deg}_{\text {out }}\left(v_{i}\right)=\sum_{j=0}^{N} a_{i j} .
$$

The degree matrix is defined as $\mathscr{D}=\operatorname{diag}\left\{d_{0}, d_{1}, \ldots, d_{N}\right\}$ with $d_{i}=\operatorname{deg}_{\text {out }}\left(v_{i}\right)$. 
Correspondingly, the Laplacian matrix is given

$$
\mathscr{L}=\mathscr{D}-\mathscr{A}=\left(\begin{array}{cc}
0 & \mathbf{0} \\
-\mathscr{A}_{0} \mathbf{1}_{N} & \mathscr{H}
\end{array}\right),
$$

where $\mathscr{H}=\mathscr{A}_{0}+\mathscr{L}_{s}$ and $\mathscr{L}_{s}=\mathscr{A}_{s}+\mathscr{D}_{s}$ with $\mathscr{D}_{s}=\operatorname{diag}\left\{d_{1}, d_{2}, \ldots, d_{N}\right\}$. Moreover, if there is one node that has directed paths to other different nodes, then the digraph $\mathscr{G}$ has a directed spanning tree.

Since the communication graph often varies in actual scenario, the switching topology is discussed in this paper. Assume that there are $l$ possible topologies, denoted as $\mathscr{G}_{\ell}$ with $\ell$ belonging to the set $\{1,2, \ldots, l\}, l \in \mathbb{Z}^{+}$. Given the time interval $\left[t_{k}, t_{k+1}\right), k \in \mathbb{N}$, where $t_{0}=0$ and $t_{k+1}-t_{k} \leq T$, there exists subintervals

$$
\left[t_{k}^{0}, t_{k}^{1}\right), \ldots,\left[t_{k}^{j}, t_{k}^{j+1}\right), \ldots,\left[t_{k}^{m_{k}-1}, t_{k}^{m_{k}}\right), t_{k}=t_{k}^{0}, t_{k+1}=t_{k}^{m_{k}},
$$

satisfying $t_{k}^{j+1}-t_{k}^{j} \geq \tau, 0 \leq j \leq m_{k}-1$ with positive dwelling time $\tau$ and positive integer $m_{k}$. The piecewise-constant switching signal $\sigma(t):[0,+\infty) \rightarrow \ell$ is defined to describe the changes in topological graphs. Obviously, $\mathscr{G}_{\sigma(t)} \in \mathscr{G}_{\ell}=\left\{\mathscr{G}_{1}, \mathscr{G}_{2}, \ldots, \mathscr{G}_{l}\right\}$. In $\left[t_{k}^{j}, t_{k}^{j+1}\right)$, the $\operatorname{graph} \mathscr{G}_{\sigma(t)}$ is fixed and denoted by $\mathscr{G}_{k j}$ and in $\left[t_{k}, t_{k+1}\right)$, some or all $\mathscr{G}_{k j}, j=0, \ldots, m_{k}-1$ can be disconnected.

For each graph $\mathscr{G}_{\sigma(t)}, \mathscr{A}_{\sigma(t)}=\left[a_{i j}^{\sigma(t)}\right] \in R^{(N+1) \times(N+1)}, \mathscr{D}_{\sigma(t)}=\left[d_{i}^{\sigma(t)}\right] \in R^{(N+1) \times(N+1)}$ and $\mathscr{L}_{\sigma(t)}=\mathscr{D}_{\sigma(t)}-\mathscr{A}_{\sigma(t)}$, respectively. Moreover, for each subgraph $\mathscr{G}_{s}^{\sigma(t)}, \mathscr{A}_{s}^{\sigma(t)}=\left[a_{i j}^{\sigma(t)}\right] \in$ $R^{N \times N}, \mathscr{D}_{s}^{\sigma(t)}=\left[d_{i}^{\sigma(t)}\right] \in R^{N \times N}$ and $\mathscr{L}_{s}^{\sigma(t)}=\mathscr{D}_{s}^{\sigma(t)}-\mathscr{A}_{s}^{\sigma(t)}$, respectively. Furthermore, $\mathscr{A}_{0}^{\sigma(t)}=\operatorname{diag}\left\{a_{10}^{\sigma(t)}, a_{20}^{\sigma(t)}, \ldots, a_{N 0}^{\sigma(t)}\right\}$ and $\mathscr{H}_{\sigma(t)}=\mathscr{A}_{0}^{\sigma(t)}+\mathscr{L}_{s}^{\sigma(t)}$.

The following assumption and lemma are key condition to ensure output regulation under switching topology.

Assumption 1. The union of the digraphs $\bigcup_{t \in\left[t_{k}, t_{k+1}\right)} \mathscr{G}_{\sigma(t)}$ has a directed spanning tree with leader as its root node.

Lemma 1. ([29]) If Assumption 1 holds, the matrix $\mathscr{H}_{\Xi}=\sum_{i=1}^{l} \mathscr{H}_{i}$ is positive definite.

\subsection{Agent's dynamics}

In this paper, the MASs containing $N$ agents are taken into account. The $i$-th agent has the following dynamics

$$
\begin{aligned}
& \dot{x}_{i}(t)=A_{i} x_{i}(t)+B_{i} u_{i}(t)+E_{i} \omega(t), \\
& y_{i}(t)=C_{i} x_{i}(t), i=1,2, \ldots, N,
\end{aligned}
$$

where $x_{i}(t) \in R^{n_{i}}$ and $y_{i}(t) \in R^{p}$ are the state and measurement output of agent $i$, separately. $u_{i}(t) \in R^{m_{i}}$ is the control protocol. And $E_{i} \omega(t)$ is the disturbance or reference input of the $i$-th agent, generated by the exosystem

$$
\begin{aligned}
& \dot{\omega}(t)=A_{0} \omega(t), \\
& y_{0}(t)=C_{0} \omega(t),
\end{aligned}
$$

where $\omega(t) \in R^{q}$ is the state of the exosystem and $y_{0}(t) \in R^{p}$ is the reference output of the $i$-th agent.

The regulation error is defined as

$$
e_{i}(t)=y_{i}(t)-y_{0}(t)=C_{i} x_{i}(t)-C_{0} \omega(t) .
$$

The main purpose of this study is to address the output regulation issue of linear heterogeneous MASs with partial unmeasurable agents under switching topology. We need to design the Luenberger observer and control protocol such that the outputs of all agents tend to the output of the exosystem, i.e., $e_{i}(t)=y_{i}(t)-y_{0}(t)=C x_{i}(t)-C_{0} \omega(t)$ $\rightarrow 0, i=1,2, \ldots, N$ as $t \rightarrow \infty$. 


\section{The design of the control protocol}

In practical occasions, not all agents' status information is measurable, thus, it is meaningful to design an observer to estimate these unmeasurable agents. In general, we assume that the states of the first $k$ agents can be measured, and the rest can not be measured. In this case, an observer that can asymptotically estimate the unmeasurable states $x_{i}, i=k+1, k+2, \ldots, N$ needs to be designed. This paper provides a full-order Luenberger observer, as follows

$$
\dot{\hat{x}}_{i}=A_{i} \hat{x}_{i}+B_{i} u_{i}+E_{i} \omega+F_{i}\left(C_{i} \hat{x}_{i}-y_{i}\right), i=k+1, k+2, \ldots, N,
$$

where the matrix $F_{i}$ is the gain matrix.

Since there are some agents that cannot receive the information from the external system, therefore, it is necessary to design the dynamic compensator to make all agents either receive information directly from the external system, or receive the compensator information of the exosystem. The dynamic compensator is designed as

$$
\dot{\eta}_{i}(t)=A_{0} \eta_{i}(t)+\rho\left(\sum_{j \in \mathscr{N}_{i}} a_{i j}^{\sigma(t)}\left(\eta_{i}(t)-\eta_{j}(t)\right)+a_{i 0}^{\sigma(t)}\left(\eta_{i}(t)-\omega(t)\right)\right),
$$

where $\rho$ is a regulation factor to be designed.

Based on the defined observer and dynamic compensator, a hybrid state feedback controller is designed as

$$
u_{i}= \begin{cases}K_{1 i} x_{i}+K_{2 i} \eta_{i} & i=1,2, \ldots, k \\ K_{1 i} \hat{x}_{i}+K_{2 i} \eta_{i} & i=k+1, k+2, \ldots, N\end{cases}
$$

where $K_{1 i} \in R^{m_{i} \times n_{i}}$ and $K_{2 i} \in R^{m_{i} \times q}$ are the gain matrices.

Let $\bar{x}=\left(x_{1}^{T}, x_{2}^{T}, \ldots, x_{k}^{T}\right)^{T}, \quad \eta=\left(\eta_{1}^{T}, \eta_{2}^{T}, \ldots, \eta_{N}^{T}\right), \quad \tilde{\omega}=\mathbf{1}_{N} \otimes \omega \quad$ and $\tilde{x}=\left(x_{k+1}^{T}, x_{k+2}^{T}, x_{N}^{T}, \hat{x}_{k+1}^{T}, \hat{x}_{k+2}^{T}, \ldots, \hat{x}_{N}^{T}\right)$. The combination of the Eqs. (1), (4) and (6) is

$$
\begin{aligned}
& \dot{\bar{x}}=\bar{A}_{o} \bar{x}+\bar{B}_{o} \eta+\bar{E}_{o} \tilde{\omega}, \\
& \dot{\tilde{x}}=\tilde{A}_{o} \tilde{x}+\tilde{B}_{o} \eta+\tilde{E}_{o} \tilde{\omega}, \\
& \dot{\eta}=\left(\rho \mathscr{H}_{\sigma(t)} \oplus A_{0}\right) \eta-\rho\left(\mathscr{A}_{0}^{\sigma(t)} \otimes I_{q}\right) \tilde{\omega},
\end{aligned}
$$

where $\bar{A}_{o}=\bar{A}+\bar{B} \bar{K}_{1}, \bar{B}_{o}=\left(\bar{B} \bar{K}_{2}, \mathbf{0}\right), \bar{E}_{o}=(\bar{E}, \mathbf{0}), \tilde{A}_{o}=\left(\begin{array}{cc}\tilde{A} & \tilde{B} \tilde{K}_{1} \\ -\tilde{F} \tilde{C} & \tilde{A}+\tilde{B} \tilde{K}_{1}+\tilde{F} \tilde{C}\end{array}\right), \tilde{B}_{o}=$ $\mathbf{1}_{2} \otimes\left(\mathbf{0}, \tilde{B} \tilde{K}_{2}\right)$, and $\tilde{E}_{o}=\mathbf{1}_{2} \otimes(\mathbf{0}, \tilde{E})$, with

$$
\begin{aligned}
& \bar{A}=\operatorname{diag}\left\{A_{1}, A_{2}, \ldots, A_{k}\right\}, \bar{B}=\operatorname{diag}\left\{B_{1}, B_{2}, \ldots, B_{k}\right\}, \bar{E}=\operatorname{diag}\left\{E_{1}, E_{2}, \ldots, E_{k}\right\}, \\
& \bar{K}_{1}=\operatorname{diag}\left\{K_{11}, K_{12}, \ldots, K_{1 k}\right\}, \bar{K}_{2}=\operatorname{diag}\left\{K_{21}, K_{22}, \ldots, K_{2 k}\right\}, \tilde{A}=\operatorname{diag}\left\{A_{k+1}, A_{k+2}, \ldots, A_{N}\right\} \\
& \tilde{B}=\operatorname{diag}\left\{B_{k+1}, B_{k+2}, \ldots, B_{N}\right\}, \tilde{C}=\operatorname{diag}\left\{C_{k+1}, C_{k+2}, \ldots, C_{N}\right\}, \tilde{E}=\operatorname{diag}\left\{E_{k+1}, E_{k+2}, \ldots, E_{N}\right\}, \\
& \tilde{F}=\operatorname{diag}\left\{F_{k+1}, F_{k+2}, \ldots, F_{N}\right\}, \tilde{K}_{1}=\operatorname{diag}\left\{K_{1(k+1)}, K_{1(k+2)}, \ldots, K_{1 N}\right\}, \\
& \tilde{K}_{2}=\operatorname{diag}\left\{K_{2(k+1)}, K_{2(k+2)}, \ldots, K_{2 N}\right\} . \\
& \quad \text { Let } \zeta=\left(\bar{x}^{T}, \tilde{x}^{T}, \eta^{T}\right)^{T} \text { and }
\end{aligned}
$$

$$
\Xi_{o}^{\sigma(t)}=\left(\begin{array}{ccc}
\bar{A}_{o} & 0 & \bar{B}_{o} \\
0 & \tilde{A}_{o} & \tilde{B}_{o} \\
0 & 0 & \rho \mathscr{H}_{\sigma(t)} \oplus A_{0}
\end{array}\right), \quad \varpi_{o}^{\sigma(t)}=\left(\begin{array}{c}
\bar{E}_{o} \\
\tilde{E}_{o} \\
-\rho\left(\mathscr{A}_{0}^{\sigma(t)} \otimes I_{q}\right)
\end{array}\right),
$$

the Eq. (7) can be rewritten as

$$
\dot{\zeta}=\Xi_{o}^{\sigma(t)} \zeta+\varpi_{o}^{\sigma(t)} \tilde{\omega} .
$$


Definition 1. The linear heterogeneous MASs (1) and (2) are said to achieve output regulation, if the hybrid control protocol (6) is designed such that the following conditions hold

(a) If $\omega(t)=0$, system (9) is asymptotically stable.

(b) The solution $\left(x_{i}(t), \omega(t)\right)$ of the following equation

$$
\begin{aligned}
& \dot{\zeta}=\Xi_{o} \zeta+\varpi_{o} \tilde{\omega}, \\
& \dot{\omega}=A_{0} \omega
\end{aligned}
$$

satisfies

$$
\lim _{t \rightarrow \infty} e_{i}(t)=\lim _{t \rightarrow \infty}\left(C_{i} x_{i}(t)-C_{0} \omega(t)\right)=0,
$$

for any initial value $x_{i}(0)$ and $\omega_{0}(0)$. Here, $\Xi_{o}=\sum_{i=1}^{l} \Xi_{o}^{i}$ and $\varpi_{o}=\sum_{i=1}^{l} \varpi_{o}^{i}$.

\section{Main results}

Before giving the main results, the following basic assumptions are first provided.

Assumption 2. The pair $\left(A_{i}, B_{i}\right)$ is stable.

Assumption 3. The pair $\left(C_{i}, A_{i}\right)$ is detectable.

Assumption 4. The eigenvalues of $A_{0}$ are in the complex left half plane.

Assumption 5. All systems satisfy the transmission zeros condition, i.e. $\operatorname{Rank}\left(\begin{array}{cc}A_{i}-\lambda I_{n_{i}} & B_{i} \\ C_{i} & \mathbf{0}\end{array}\right)=n_{i}+p$ for $\forall \lambda \in \sigma\left(A_{0}\right)$.

Lemma 2. (Cauchy's Convergence Criterion [30]): For arbitrary $\varepsilon>0$, there is $M_{\varepsilon} \in \mathbb{Z}^{+}$ such that for arbitrary $k>M_{\varepsilon},\left|V\left(t_{k+1}\right)-V\left(t_{k}\right)\right|<\varepsilon$, then the sequence $V\left(t_{k}\right), k=0,1,2, \ldots$ converges.

Theorem 3. The matrices $\bar{A}_{o}$ and $\tilde{A}_{o}$ (defined in Eq. (7)) are Hurwitz if Assumptions 2 and 3 hold.

Proof. If the matrix pair $\left(A_{i}, B_{i}\right)$ is stabilizable and $\left(C_{i}, A_{i}\right)$ is detectable, then there exist matrices $K_{1 i}$ and $F_{i}$ such that $A_{i}+B_{i} K_{1 i}$ and $A_{i}+F_{i} C_{i}$ are Hurwitz. It is obvious that $\bar{A}_{o}=\bar{A}+\bar{B} \bar{K}_{1}$ is Hurwitz when $A_{i}+B_{i} K_{1 i}$ is Hurwitz.

Let

$$
S=\left(\begin{array}{ll}
\mathbf{I} & \mathbf{0} \\
\mathbf{I} & \mathbf{I}
\end{array}\right), \quad \tilde{A}_{o s}=\left(\begin{array}{cc}
\tilde{A}+\tilde{B} \tilde{K}_{1} & \tilde{B} \tilde{K}_{1} \\
\mathbf{0} & \tilde{A}+\tilde{F} \tilde{C}
\end{array}\right),
$$

then we have $\tilde{A}_{o}=S \tilde{A}_{o s} S^{-1}$. Hence, $\tilde{A}_{o}$ is Hurwitz if and only if $\tilde{A}_{o s}$ is Hurwitz. $\tilde{A}_{o s}$ is Hurwitz when $\tilde{A}+\tilde{B} \tilde{K}_{1}$ and $\tilde{A}+\tilde{F} \tilde{C}$ are Hurwitz simultaneously. Moreover, $\tilde{A}+\tilde{B} \tilde{K}_{1}$ and $\tilde{A}+\tilde{F} \tilde{C}$ are Hurwitz when $A_{i}+B_{i} K_{1 i}$ and $A_{i}+F_{i} C_{i}$ are Hurwitz. This completes the proof.

Theorem 4. If Assumptions 1-3 are satisfied, and there are matrices $\Pi_{i}$ and $\Gamma_{i}$ satisfying the following regulator equations:

$$
\begin{aligned}
& \Pi_{i} A_{0}=A_{i} \Pi_{i}+B_{i} \Gamma_{i}+E_{i}, \\
& 0=C_{i} \Pi_{i}-C_{0}
\end{aligned}
$$

with $\sigma\left(A_{0}\right) \subset j \boldsymbol{R}$, then the output regulation issue can be addressed by the hybrid feedback controller (6). 
Proof. Do the following coordinate transformation

$$
\begin{aligned}
& \chi_{i}=x_{i}-\Pi_{i} \omega, i=1,2, \ldots, k, \\
& \hat{\chi}_{i}=\hat{x}_{i}-\Pi_{i} \omega, i=k+1, k+2, \ldots, N,
\end{aligned}
$$

where $\Pi_{i}$ is the solution of Eq. (11).

Let $\bar{\chi}=\left(\chi_{1}^{T}, \chi_{2}^{T}, \ldots, \chi_{k}^{T}\right)$ and $\tilde{\chi}=\left(\chi_{k+1}^{T}, \chi_{k+2}^{T}, \ldots, \chi_{N}^{T}, \hat{\chi}_{k+1}^{T}, \hat{\chi}_{k+2}^{T}, \ldots, \hat{\chi}_{N}^{T}\right)$, then

$$
\bar{\chi}=\bar{x}-(\bar{\Pi}, \mathbf{0}) \tilde{\omega}, \quad \tilde{\chi}=\tilde{x}-\mathbf{1}_{2} \otimes(\mathbf{0}, \tilde{\Pi}) \tilde{\omega},
$$

where $\bar{\Pi}=\operatorname{diag}\left\{\Pi_{1}, \Pi_{2}, \ldots, \Pi_{k}\right\}$, and $\tilde{\Pi}=\operatorname{diag}\left\{\Pi_{k+1}, \Pi_{k+2}, \ldots, \Pi_{N}\right\}$.

The derivative of Eq. (12) is given as

$$
\dot{\bar{\chi}}=\dot{\bar{x}}-(\bar{\Pi}, \mathbf{0}) \dot{\tilde{\omega}}, \quad \dot{\tilde{\chi}}=\dot{\tilde{x}}-\mathbf{1}_{2} \otimes(\mathbf{0}, \tilde{\Pi}) \dot{\tilde{\omega}} .
$$

By using $\dot{\tilde{\omega}}=\mathbf{1}_{N} \otimes A_{0} \omega=\left(I_{N} \otimes A_{0}\right) \tilde{\omega}$ and combining the Eqs. (2), (7), (12) and (13), one has

$$
\begin{aligned}
& \dot{\bar{\chi}}=\bar{A}_{o} \bar{\chi}+\bar{B}_{o} \eta+\bar{E}_{\chi_{o}} \tilde{\omega}, \\
& \dot{\tilde{\chi}}=\tilde{A}_{o} \tilde{\chi}+\tilde{B}_{0} \eta+\tilde{E}_{\chi_{o}} \tilde{\omega},
\end{aligned}
$$

where

$$
\begin{aligned}
& \bar{E}_{\chi o}=\bar{A}_{o}(\bar{\Pi}, \mathbf{0})+\bar{E}_{o}-(\bar{\Pi}, \mathbf{0})\left(I_{N} \otimes A_{0}\right), \\
& \tilde{E}_{\chi_{o}}=\tilde{A}_{o}\left(\mathbf{1}_{2} \otimes(\mathbf{0}, \tilde{\Pi})\right)+\tilde{E}_{o}-\left(\mathbf{1}_{2} \otimes(\mathbf{0}, \tilde{\Pi})\right)\left(I_{N} \otimes A_{0}\right) .
\end{aligned}
$$

Let the gain matrix $K_{2 i}$ be $K_{2 i}=\Gamma_{i}-K_{1 i} \Pi_{i}$, the first equation of Eq. (11) can be rewritten as

$$
\Pi_{i} A_{0}=\left(A_{i}+B_{i} K_{1 i}\right) \Pi_{i}+B_{i} K_{2 i}+E_{i},
$$

then Eq. (16) can be changed into

$$
\begin{aligned}
(\bar{\Pi}, \mathbf{0})\left(I_{N} \otimes A_{0}\right) & =\left(\bar{\Pi}\left(I_{k} \otimes A_{0}\right), \mathbf{0}\right) \\
& =\left(\left(\bar{A}+\bar{B} \bar{K}_{1}\right) \bar{\Pi}+\bar{B}_{K_{2}}+\bar{E}, \mathbf{0}\right) \\
& =\bar{A}_{o}(\bar{\Pi}, \mathbf{0})+\bar{B}_{o}+\bar{E}_{o} .
\end{aligned}
$$

Since

$$
\begin{aligned}
\mathbf{1}_{2} \otimes\left(\mathbf{0},\left(\tilde{A}+\tilde{B} \tilde{K}_{1}\right) \tilde{\Pi}\right) & =\left(\begin{array}{cc}
\mathbf{0} & \left(\tilde{A}+\tilde{B} \tilde{K}_{1}\right) \tilde{\Pi} \\
\mathbf{0} & \left(\tilde{A}+\tilde{B} \tilde{K}_{1}\right) \tilde{\Pi}
\end{array}\right) \\
& =\left(\begin{array}{cc}
\tilde{A} & \tilde{B} \tilde{K}_{1} \\
-\tilde{F} \tilde{C} & \tilde{A}+\tilde{B} \tilde{K}_{1}+\tilde{F} \tilde{C}
\end{array}\right)\left(\begin{array}{cc}
\mathbf{0} & \tilde{\Pi} \\
\mathbf{0} & \tilde{\Pi}
\end{array}\right) \\
& =\tilde{A}_{o}\left(\mathbf{1}_{2} \otimes(\mathbf{0}, \tilde{\Pi})\right),
\end{aligned}
$$

the Eq. (16) also has a form

$$
\begin{aligned}
\left(\mathbf{1}_{2} \otimes(\mathbf{0}, \tilde{\Pi})\right)\left(I_{N} \otimes A_{0}\right) & =\mathbf{1}_{2} \otimes\left(\mathbf{0}, \tilde{\Pi}\left(I_{N-k} \otimes A_{0}\right)\right) \\
& =\mathbf{1}_{2} \otimes\left(\mathbf{0},\left(\tilde{A}+\tilde{B} \tilde{K}_{1}\right) \tilde{\Pi}+\tilde{B} \tilde{K}_{2}+\tilde{E}\right) \\
& =\tilde{A}_{o}\left(\mathbf{1}_{2} \otimes(\mathbf{0}, \tilde{\Pi})\right)+\tilde{B}_{o}+\tilde{E}_{o} .
\end{aligned}
$$

Combining the Eqs. (15), (17) and (18) yields $\bar{E}_{\chi_{o}}=-\bar{B}_{o}$ and $\tilde{E}_{\chi_{o}}=-\tilde{B}_{o}$. Thus the Eq. (14) can be expressed as

$$
\begin{aligned}
& \dot{\bar{\chi}}=\bar{A}_{o} \bar{\chi}+\bar{B}_{o}(\eta-\tilde{\omega}), \\
& \dot{\tilde{\chi}}=\tilde{A}_{o} \tilde{\chi}+\tilde{B}_{o}(\eta-\tilde{\omega}) .
\end{aligned}
$$

Taking the derivative of $\eta-\tilde{\omega}$, we have

$$
\begin{aligned}
\dot{\eta}-\dot{\tilde{\omega}} & =\left(\rho \mathscr{H}_{\sigma(t)} \oplus A_{0}\right) \eta-\rho\left(\mathscr{A}_{0}^{\sigma(t)} \otimes I_{q}\right) \tilde{\omega}-\left(I_{N} \otimes A_{0}\right) \tilde{\omega} \\
& =\left(\rho \mathscr{H}_{\sigma(t)} \oplus A_{0}\right) \eta-\left(\rho\left(\mathscr{H}_{\sigma(t)} \otimes I_{q}\right)+\left(I_{N} \otimes A_{0}\right)\right) \tilde{\omega} \\
& =\left(\rho \mathscr{H}_{\sigma(t)} \oplus A_{0}\right)(\eta-\tilde{\omega}) .
\end{aligned}
$$


Cai, Y. L. et al.

Let $\xi=\left(\bar{\chi}^{T}, \tilde{\chi}^{T},(\eta-\tilde{\omega})^{T}\right)^{T}$, the combination of Eqs. (19) and (20) gives

$$
\dot{\xi}=\Xi_{o}^{\sigma(t)} \xi,
$$

where $\Xi_{o}^{\sigma(t)}$ is given in Eq. (8). The eigenvalues of matrix $\rho \mathscr{H}_{\sigma(t)} \oplus A_{0}$ are expressed as

$$
\lambda\left(\rho \mathscr{H}_{\sigma(t)} \oplus A_{0}\right)=\left\{\rho \lambda_{i}\left(\mathscr{H}_{\sigma(t)}\right)+\lambda_{j}\left(A_{0}\right) \mid i=1,2, \ldots, N \text {, and } j=1,2, \ldots, q\right\} .
$$

Obviously, there is $\rho<0$ satisfying that $\Xi_{o}^{\sigma(t)}$ is negative semi-definite. Taking the Lyapunov function candidate as

$$
V=\frac{1}{2} \xi^{T} \xi
$$

the derivative of $V$ is

$$
\dot{V}=\xi^{T} \Xi_{o}^{\sigma(t)} \xi .
$$

Combining $\dot{V} \leq 0$ with $V \geq 0$, one can obtain that $V$ is bounded and $\lim _{t \rightarrow \infty} V(t)$ exists. By the Cauchy's convergence criterion in Lemma 2, for arbitrary $\varepsilon>0$, there is $M_{\varepsilon} \in \mathbb{Z}^{+}$such that for arbitrary $k>M_{\mathcal{E}}$,

$$
\left|V\left(t_{k+1}\right)-V\left(t_{k}\right)\right|<\varepsilon
$$

or

$$
\left|\int_{t_{k}}^{t_{k}+1} \dot{V}(t)\right|<\varepsilon
$$

It follows that

$$
\left|\int_{t_{k}^{0}}^{t_{k}^{1}} \dot{V}(t)\right|+\left|\int_{t_{k}^{1}}^{t_{k}^{2}} \dot{V}(t)\right|+\ldots+\left|\int_{t_{k}}^{t_{k}^{m_{k}}} \dot{m_{k}} \dot{V}(t)\right|<\varepsilon .
$$

In light of (23), in each time subinterval $\left[t_{k}^{j}, t_{k}^{j+1}\right), j=0,1, \ldots, m_{k}-1$, we have

$$
\left|\int_{t_{k}^{j}}^{t_{k}^{j+1}} \dot{V}(t)\right|=\left|\int_{t_{k}^{j}}^{t_{k}^{j+1}} \xi^{T} \Xi_{o}^{\sigma\left(t_{k}^{j}\right)} \xi\right| \geq\left|\int_{t_{k}^{j}}^{t_{k}^{j}+\tau} \xi^{T} \Xi_{o}^{\sigma\left(t_{k}^{j}\right)} \xi\right| .
$$

Combining (24) with (25) gives

$$
\varepsilon>\left|\int_{t_{k}^{0}}^{t_{k}^{0}+\tau} \xi^{T} \Xi_{o}^{\sigma\left(t_{k}^{0}\right)} \xi\right|+\ldots+\left|\int_{t_{k}^{m} m^{m_{k}-1}}^{t_{k} k^{-1}+\tau} \xi^{T} \Xi_{o}^{\sigma\left(t_{k}^{m_{k}-1}\right)} \xi\right|,
$$

that is, for any $k>M_{\varepsilon}$

$$
\left|\int_{t_{k}^{j}}^{t_{k}^{j}+\tau} \xi^{T} \Xi_{o}^{\sigma\left(t_{k}^{j}\right)} \xi\right|<\varepsilon
$$

From (27), one has

$$
\lim _{t \rightarrow \infty} \int_{t}^{t+\tau} \xi(s)^{T} \Xi_{o}^{\sigma\left(t_{k}^{j}\right)} \xi(s) d s=0 .
$$

Since only finite switches take place during $\left[t_{k}, t_{k+1}\right)$, we get

$$
\lim _{t \rightarrow \infty} \int_{t}^{t+\tau}\left\{\xi(s)^{T} \Xi_{o}^{\sigma\left(t_{k}^{0}\right)} \xi(s)+\ldots+\xi(s)^{T} \Xi_{o}^{\sigma\left(t_{k}^{m_{k}-1}\right)} \xi(s)\right\} d s=0
$$

which can be expressed as

$$
\lim _{t \rightarrow \infty} \int_{t}^{t+\tau} \xi(s)^{T} \Xi_{o} \xi(s) d s=0,
$$


where $\Xi_{o}=\Xi_{o}^{\sigma\left(t_{k}^{0}\right)}+\ldots+\Xi_{o}^{\sigma\left(t_{k}^{m_{k}-1}\right)}=\left(\begin{array}{ccc}l \bar{A}_{o} & 0 & l \bar{B}_{o} \\ 0 & l \tilde{A}_{o} & l \tilde{B}_{o} \\ 0 & 0 & \rho \mathscr{H}_{\Xi} \oplus A_{0}\end{array}\right), \mathscr{H}_{\Xi}=\sum_{i=1}^{l} \mathscr{H}_{i}$.

According to Assumption 1 and Lemma $1, \bigcup_{t \in\left[t_{k}, t_{k+1}\right)} \mathscr{G}_{\sigma(t)}$ has a spanning tree, thus $\mathscr{H}_{\Xi}$ is positive definite. The eigenvalues of $\rho \mathscr{H} \Xi \oplus A_{0}$ are given as

$$
\lambda\left(\rho \mathscr{H} \Xi \oplus A_{0}\right)=\left\{\rho \lambda_{i}\left(\mathscr{H}_{\Xi}\right)+\lambda_{j}\left(A_{0}\right) \mid i=1,2, \ldots, N, j=1,2, \ldots, q\right\} .
$$

There is $\rho<0$ satisfying that all eigenvalues of $\rho \mathscr{H} \Xi \oplus A_{0}$ are in the complex left half plane, i.e., $\rho \mathscr{H} \Xi \oplus A_{0}$ is Hurwitz. By the Theorem $1, \Xi_{o}$ is Hurwitz, then we have $\lim _{t \rightarrow \infty} \xi=0$, i.e., $\lim _{t \rightarrow \infty} \chi_{i}=0$.

Substituting $\chi_{i}=x_{i}-\prod_{i} \omega$ into Eq. (3) yields

$$
\begin{aligned}
e_{i} & =C\left(\chi_{i}+\Pi_{i} \omega\right)-C_{0} \omega \\
& =C \chi_{i}+\left(C \Pi_{i}-C_{0}\right) \omega \\
& =C \chi_{i},
\end{aligned}
$$

then $\lim _{t \rightarrow \infty} e_{i}=\lim _{t \rightarrow \infty} C \chi_{i}=0$. Therefore, the conclusion is proved.

The process to design the hybrid feedback control strategy (6) is presented in Algorithm 1.

Algorithm 1. Assuming that the Assumptions 1-3 are satisfied, then the hybrid feedback control strategy (6) can be designed as follows

1. Choose the gain matrices $K_{1 i}$ and $F_{i}$ such that $A_{i}+B_{i} K_{1 i}$ and $A_{i}+F_{i} C_{i}$ are Hurwitz.

2. Solve the regulator equations (11) to get $\Pi_{i}$ and $\Gamma_{i}$, then the gain matrix $K_{2 i}=$ $\Gamma_{i}-K_{1 i} \Pi_{i}$ can be obtained.

3. Choose $\rho<0$ such that $\Xi_{o}^{\sigma(t)}$ defined in (8) is negative semi-definite.

Here, the gain matrices $K_{1 i}, K_{2 i}, F_{i}$ and regulation factor $\rho$ are the parameters of the control method (6).

Remark 1 . Let $x_{i}^{*}$ be the difference between the system variable $x_{i}$ and the estimated variable $\hat{x}_{i}$, then

$$
\begin{aligned}
\dot{x}_{i}^{*} & =\dot{x}_{i}-\dot{\hat{x}}_{i} \\
& =A_{i} x_{i}+B_{i} u_{i}+E_{i} \omega-\left(A_{i} \hat{x}_{i}+B_{i} u_{i}+E_{i} \omega+F_{i}\left(C_{i} \hat{x}_{i}-C_{i} x_{i}\right)\right) \\
& =\left(A_{i}+F_{i} C_{i}\right) x_{i}^{*} .
\end{aligned}
$$

Because the pair $\left(C_{i}, A_{i}\right)$ is detectable, so there exists matrix $F_{i}$ such that $A_{i}+F_{i} C_{i}$ is Hurwitz, i.e., $x_{i}^{*} \rightarrow 0,(t \rightarrow \infty)$.

Remark 2. In this paper, we consider the partial unmeasurable agents, i.e. not all agents are measurable or unmeasurable. We only design the observer for the unmeasurable agents. For the unmeasurable agents, we can design the reduced-order observer by introducing the state transformation $x_{i}(t)=\mathscr{T}_{i} \bar{x}_{i}(t), i=k+1, \ldots, N$, then the system (1) can be changed into

$$
\left\{\begin{array}{l}
\dot{x}_{m i}(t)=A_{i}^{11} x_{m i}(t)+A_{i}^{12} x_{u i}(t)+B_{i}^{1} u_{i}(t)+E_{i}^{1} \omega(t) \\
\dot{x}_{u i}(t)=A_{i}^{21} x_{m i}(t)+A_{i}^{22} x_{u i}(t)+B_{i}^{2} u_{i}(t)+E_{i}^{2} \omega(t) \\
y_{i}(t)=x_{m i}(t), i=k+1, \ldots, N
\end{array}\right.
$$

where $\hat{A}_{i}=\mathscr{T}_{i}^{-1} A_{i} \mathscr{T}_{i}=\left(\begin{array}{cc}A_{i}^{11} & A_{i}^{12} \\ A_{i}^{21} & A_{i}^{22}\end{array}\right), \hat{B}_{i}=\mathscr{T}_{i}^{-1} B_{i}=\left(\begin{array}{c}B_{i}^{1} \\ B_{i}^{2}\end{array}\right), \hat{C}_{i}=C_{i} \mathscr{T}_{i}=\left(I_{p}, \mathbf{0}\right)$ and $\hat{E}_{i}=\mathscr{T}_{i}^{-1} E_{i}=\left[E_{i}^{1}, E_{i}^{2}\right]^{\prime}$. The reduced-order observer is designed as

$$
\tilde{x}_{u i}(k+1)=A_{i}^{21} x_{m i}(k)+A_{i}^{22} \tilde{x}_{u i}(k)+B_{i}^{2} u_{i}(k)+E_{i}^{2} w(k)+L_{i} A_{i}^{12}\left(x_{u i}(k)-\tilde{x}_{u i}(k)\right),
$$


where $L_{i}$ is a constant matrix. And the distributed feedback controller can be designed as

$$
\begin{aligned}
& \delta_{i}(t)=\sum_{j \in N_{i}} a_{i j}\left(C_{i} x_{i}(t)-C_{j} x_{j}(t)\right)+a_{i 0}\left(C_{i} x_{i}(t)-y_{r i}(t)\right) \\
& \xi_{i}(t)=\sum_{j \in N_{i}} a_{i j}\left(\tilde{x}_{u i}(t)-\tilde{x}_{u j}(t)\right)+a_{i 0} \tilde{x}_{t i}(t) \\
& \dot{\eta}_{i}(t)=G_{1} \eta_{i}(t)+G_{2} \delta_{i}(t) \\
& u_{i}(t)=K_{1 i} \xi_{i}(t)+K_{2 i} \delta_{i}(t)+K_{3 i} \eta_{i}(t), i=k+1, \ldots, N
\end{aligned}
$$

where $K_{1 i} \in R^{q \times(n-p)}, K_{2 i} \in R^{q \times p}$ and $K_{3 i} \in R^{q \times s_{m}}$ are the gain matrices. $\theta_{i}>0$ is a parameter to be designed. And the matrix pair $\left(G_{1}, G_{2}\right)$ is the $p$-copy internal model. The proof process is similar to that in [31]. Note that in above controller, the dimensions of unmeasurable states for all agents are assumed to be the same.

\section{Simulation and examples}

To illustrate the theoretical results, we take the linear heterogeneous MASs with five agents indexed by $0-4$ into account. The node 0 denotes the exosystem, and the nodes $1-4$ denote the four agents. Three cases including 1) partial unmeasurable agents 2) all measurable agents and 3) all unmeasurable agents are considered.

\subsection{Numerical examples}

Example 1. In this example, we take the linear heterogeneous MASs with the same dimensions into account. The four agents are expressed as

$$
\begin{aligned}
& \dot{x}_{i}(t)=A_{i} x_{i}(t)+B_{i} u_{i}(t)+E_{i} \omega(t), \\
& y_{i}(t)=C_{i} x_{i}(t), i=1,2,3,4,
\end{aligned}
$$

where $A_{i}=\left(\begin{array}{cc}0 & 1 \\ 0 & -1\end{array}\right), B_{i}=\left(\begin{array}{c}0 \\ 1\end{array}\right), C_{i}=[1,0]$ and $E_{i}=\left(\begin{array}{cc}0 & 0 \\ 0 & -i\end{array}\right)$.

The dynamics of the exosystem are described as

$$
\begin{aligned}
& \dot{\omega}(t)=A_{0} \omega(t), \\
& y_{0}(t)=C_{0} \omega(t),
\end{aligned}
$$

where $A_{0}=\left(\begin{array}{cc}0 & 1 \\ -1 & 0\end{array}\right)$ and $C_{0}=[1,0]$.

Four switching topologies $\mathscr{G}_{1}, \mathscr{G}_{2}, \mathscr{G}_{3}$ and $\mathscr{G}_{4}$ are considered in this paper, shown in Fig. 1. Suppose that the interaction topologies switch as $\mathscr{G}_{1} \rightarrow \mathscr{G}_{2} \rightarrow \mathscr{G}_{3} \rightarrow \mathscr{G}_{4} \rightarrow \mathscr{G}_{1} \rightarrow \ldots$. And the dwelling time is selected as $\tau=0.5 \mathrm{~s}$.

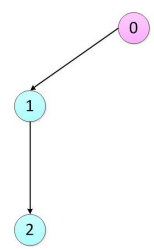

(a)

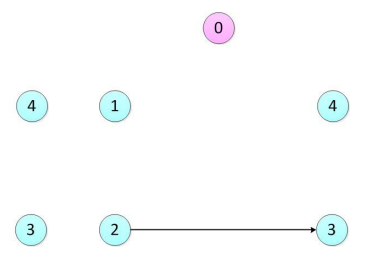

(b)
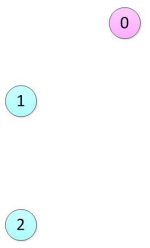

(c)

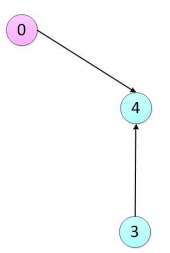

(d)

Figure 1. Communication topologies: (a) $\mathscr{G}_{1}$, (b) $\mathscr{G}_{2}$, (c) $\mathscr{G}_{3}$, and (d) $\mathscr{G}_{4}$

By the regulator equations (11), the matrices $\Pi_{i}=\left(\begin{array}{ll}1 & 0 \\ 0 & 1\end{array}\right)(i=1,2,3,4), \Gamma_{1}=$ $[-1,2], \Gamma_{2}=[-1,3], \Gamma_{3}=[-1,4]$ and $\Gamma_{4}=[-1,5]$ can be obtained. Since the pair $\left(A_{i}, B_{i}\right)$ is stabilizable, there exists matrix $K_{1 i}=[-6,-6]$ such that $A_{i}+B_{i} K_{1 i}(i=1,2,3,4)$ is Hurwitz. 
Cai, Y. L. et al.

Then $K_{21}=\Gamma_{1}-K_{11} \Pi_{1}=[5,8], K_{22}=[5,9], K_{23}=[5,10]$ and $K_{24}=[5,11]$. Besides, the pair $\left(C_{i}, A_{i}\right)$ is detectable, there exists matrix $F_{i}=[-8 ;-8]$ such that $A_{i}+F_{i} C_{i}(i=1,2,3,4)$ is Hurwitz. Moreover, the regulation factor $\rho=-1$.

The experiment is conducted with random initial states. The simulation results are given in Figs. 2-9. For case 1, the agents 1, 2 are assumed to be measurable, i.e. $k=2$. Fig. 2 depicts the measurement outputs of all agents. It can be observed that the trajectories of the measurement outputs are consistent with that of the reference output. The regulation errors are shown in Fig. 3. One can get that all regulation errors converge to zero asymptotically. Besides, we use $e_{i}^{*}=\sqrt{\left\|x_{i}(t)-\hat{x}_{i}(t)\right\|^{2}}, i=3,4$ to denote the estimation errors of MASs (24) under the designed observer (4). Fig. 4 presents these results. It demonstrates that the designed observer (4) can estimate the unmeasurable states $x_{3}$ and $x_{4}$ very well. For case 2 , the obtained results are presentd in Figs. 5 and 6. We can get that the regulation errors of four measurable agents tend to zero asymptotically. And if $k=0$, the numerical results for case 3 (i.e., all agents' states are unmeasurable) are shown in Figs. 7 and 8. We can get that the regulation errors of four unmeasurable agents can tend to zero. And the estimation errors of all unmeasurable agents are depicted in Fig. 9, which indicates that the estimation errors can tend to zero. From the above three cases, we can get that whether the states are measurable or not, the control protocol (6) can settle the output regulation issue of linear heterogeneous MASs under switching topology.

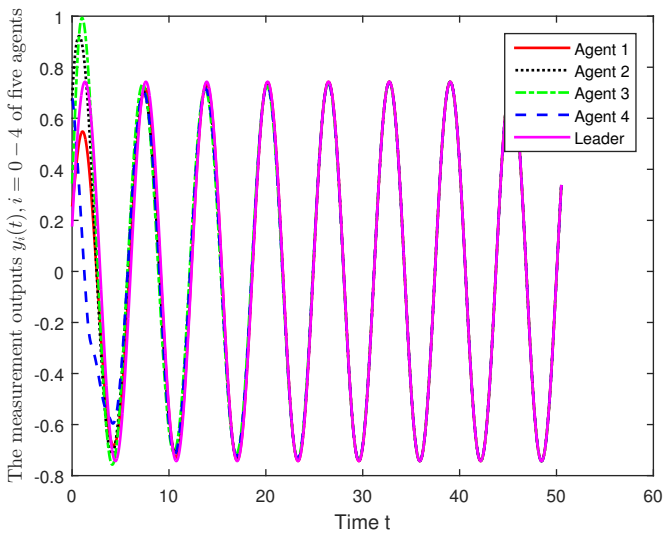

Figure 2. Example 1: the measurement outputs $y_{i}(t)$ of five agents; the agents 3, 4 are unmeasurable

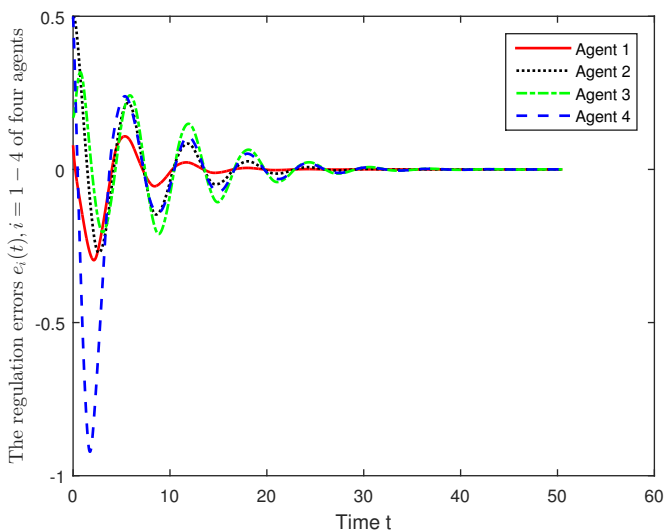

Figure 3. Example 1: the regulation errors $e_{i}(t)$ of four agents; the agents 3, 4 are unmeasurable 
Cai, Y. L. et al.

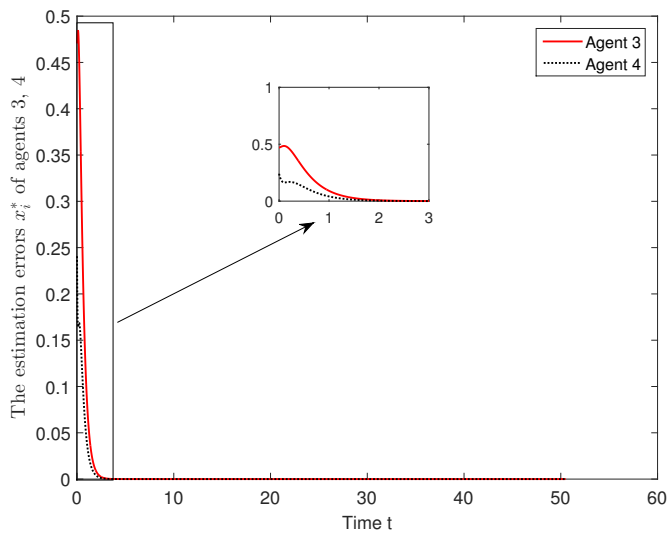

Figure 4. Example 1: the estimation errors $x_{i}^{*}$ of agents 3 and 4

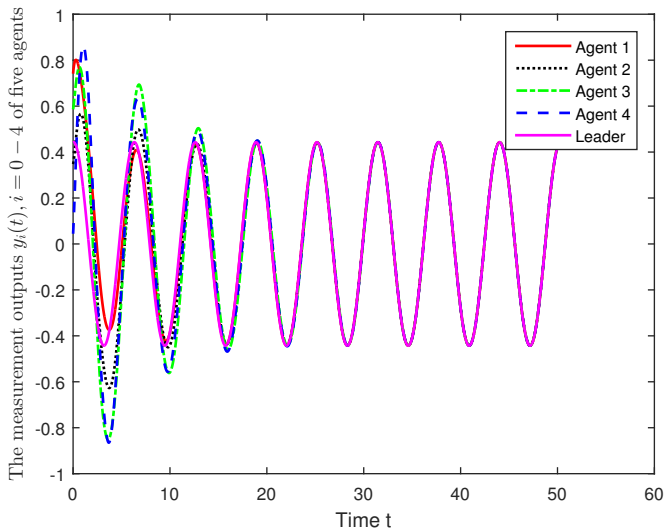

Figure 5. Example 1: the measurement outputs $y_{i}(t)$ of five agents

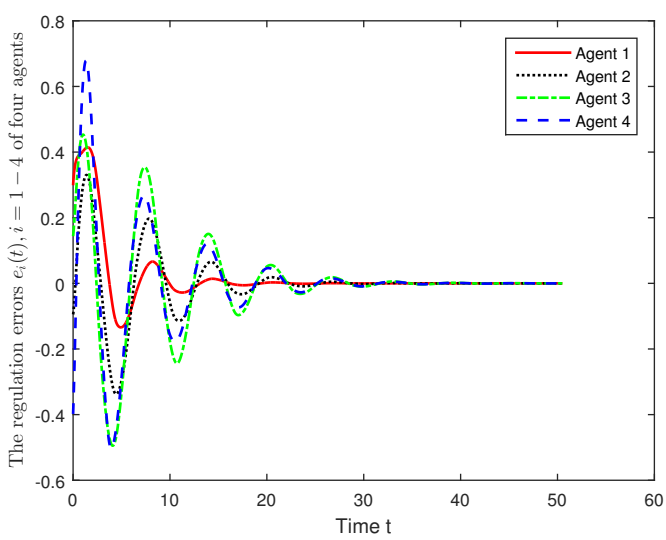

Figure 6. Example 1: the regulation errors $e_{i}(t)$ of four measurable agents

Example 2. In this example, we take the linear heterogeneous MASs with different dimensions into consideration. The dynamics and output matrices of agents 1,2 and the exosystem are the same as those of Example 1. The dynamics and output matrices of agents 
Cai, Y. L. et al.

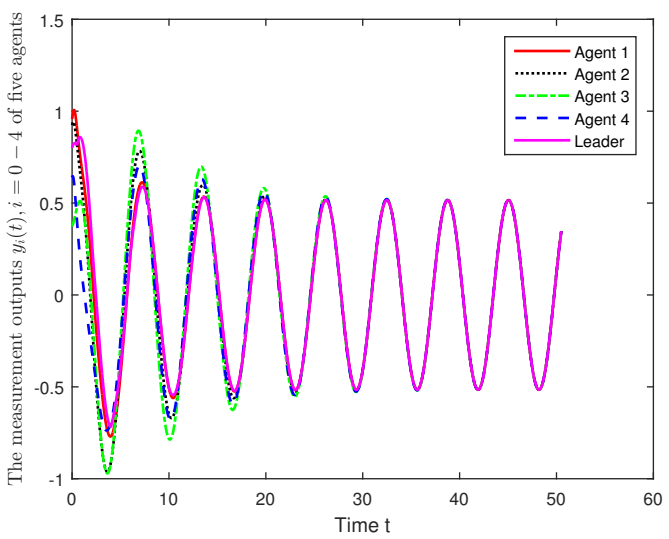

Figure 7. Example 1: the measurement outputs $y_{i}(t)$ of five agents

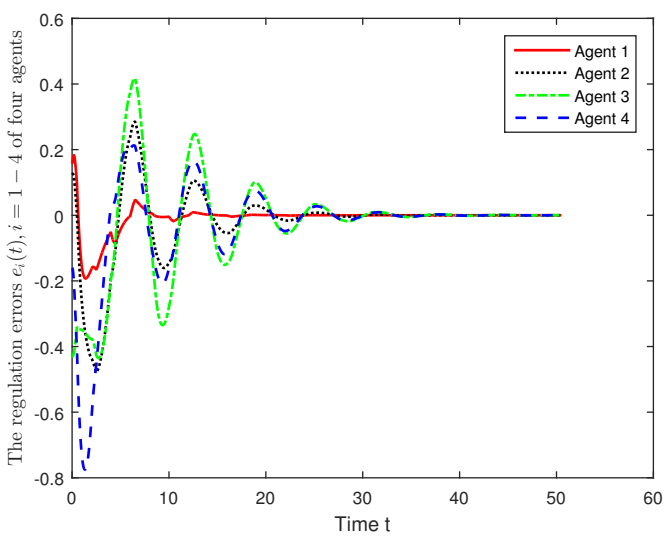

Figure 8. Example 1: the regulation errors $e_{i}(t)$ of four unmeasurable agents

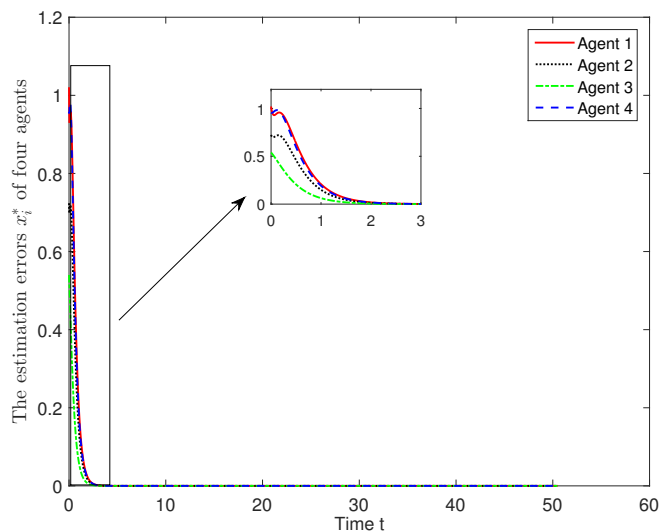

Figure 9. Example 1: the estimation errors $x_{i}^{*}$ of four unmeasurable agents

3,4 are described as (30) with $A_{i}=\left(\begin{array}{ccc}0 & 1 & 0 \\ 0 & 0 & 1 \\ 0 & -1 & -2\end{array}\right), B_{i}=\left(\begin{array}{c}0 \\ 0 \\ 1\end{array}\right), C_{i}=[1,0,0]$ and 
Cai, Y. L. et al.

$E_{i}=\left(\begin{array}{cc}0 & 0 \\ 0 & 0 \\ 0 & -i\end{array}\right)(i=3,4)$. The matrices $\Pi_{i}, \Gamma_{i}, K_{1 i}$ and $F_{i}$ for $i=1,2$ and the regulation factor $\rho$ are the same as those of Example 1. The matrices $\Pi_{i}=\left(\begin{array}{cc}1 & 0 \\ 0 & 1 \\ -1 & 0\end{array}\right)(i=3,4)$, $\Gamma_{3}=[-2,3]$ and $\Gamma_{4}=[-2,4]$. Besides, $K_{1 i}=[-6,-6,-6], F_{i}=[-8 ;-8 ;-8](i=3,4)$, $K_{23}=[-2,9]$ and $K_{24}=[-2,10]$.

Similar to Example 1, the experiment is also conducted with random initial states. The simulation results are given in Figs. 10-17. For case 1, assume $k=2$, the measurement outputs and regulation errors are depicted in Figs. 10 and 11. From Fig. 10, we can get that the output trajectories of four agents can track that of the external system. Fig. 11 indicates that the regulation errors of four agents converge to zero asymptotically. Moreover, the estimation errors of agents 3 and 4 are shown in Fig. 12. It demonstrates that the designed observer (4) can asymptotically estimate the unmeasurable states $x_{3}$ and $x_{4}$. For case 2, i.e. $k=4$, the simulation results are shown in Figs. 13 and 14. It can be observed that the regulation errors of four measurable agents asymptotically converge to zero. For case 3, i.e. $k=0$, the measurement outputs and regulation errors of all agents are presented in Figs. 15 and 16 . We can get that the regulation errors of four unmeasurable agents can tend to zero. Moreover, the estimation errors of all agents are depicted in Fig. 17. From the above results, we can see that the proposed controller (6) can address the output regulation issue of linear heterogeneous MASs under switching topology.

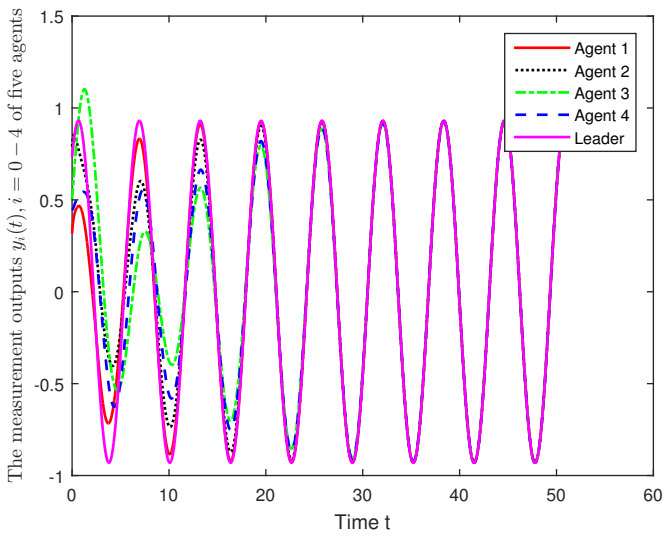

Figure 10. Example 2: the measurement outputs $y_{i}(t)$ of five agents; the agents 3, 4 are unmeasurable

\subsection{Comparison example}

In this section, the comparison method in [17] is used for illustrating the merits of our controller. Moreover, the directed graph in [17] is used for comparison. The system matrices are given as follows $A_{1}=\left(\begin{array}{ll}0 & 1 \\ 0 & 0\end{array}\right), A_{2}=\left(\begin{array}{cc}0 & 1 \\ -1 & 0\end{array}\right), A_{3}=\left(\begin{array}{cc}0 & -1 \\ 1 & 0\end{array}\right), A_{4}=\left(\begin{array}{cc}0 & -1 \\ 0 & 0\end{array}\right), A 5=A 6=$ $A 7=A 8=A 9=A 4, B_{1}=\left(\begin{array}{l}0 \\ 1\end{array}\right), B_{2}=\left(\begin{array}{l}1 \\ 1\end{array}\right), B_{3}=\left(\begin{array}{l}0 \\ 1\end{array}\right), B_{4}=\left(\begin{array}{l}0 \\ 1\end{array}\right), B 5=B 6=$ $B 7=B 8=B 9=B 4, E_{1}=\left(\begin{array}{cc}0 & 0 \\ 1 & -0.5\end{array}\right), E_{2}=\left(\begin{array}{cc}-1 & 0.5 \\ -1 & 0.5\end{array}\right), E_{3}=\left(\begin{array}{cc}0 & 2 \\ -1 & 0\end{array}\right), E_{4}=$ $\left(\begin{array}{cc}0 & 2 \\ -1 & 1\end{array}\right), E 5=E 6=E 7=E 8=E 9=E 4$, and $C_{i}=[1,0], i=1,2, \ldots, 9$. The 
Cai, Y. L. et al.

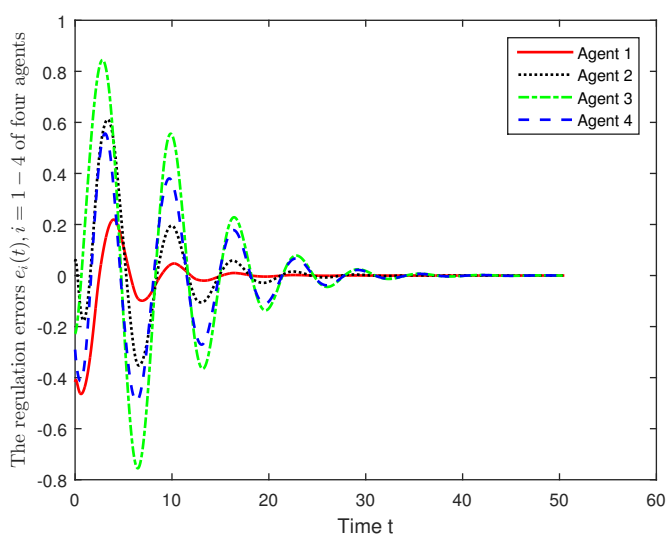

Figure 11. Example 2: the regulation errors $e_{i}(t)$ of four agents; the agents 3, 4 are unmeasurable

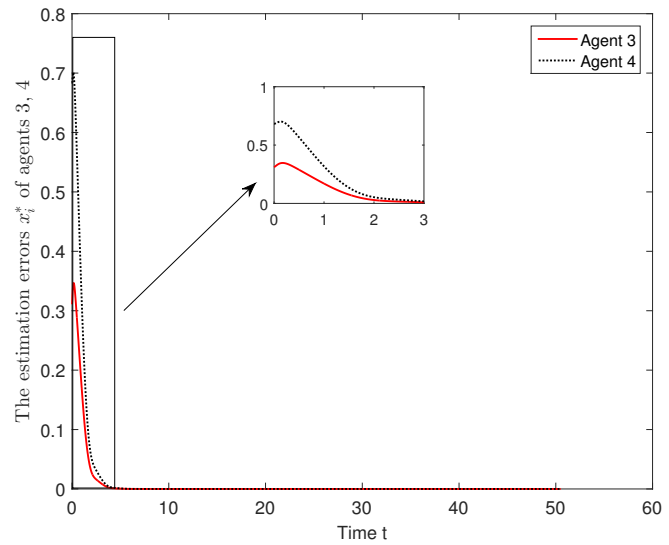

Figure 12. Example 2: the estimation errors $x_{i}^{*}$ of agents 3 and 4

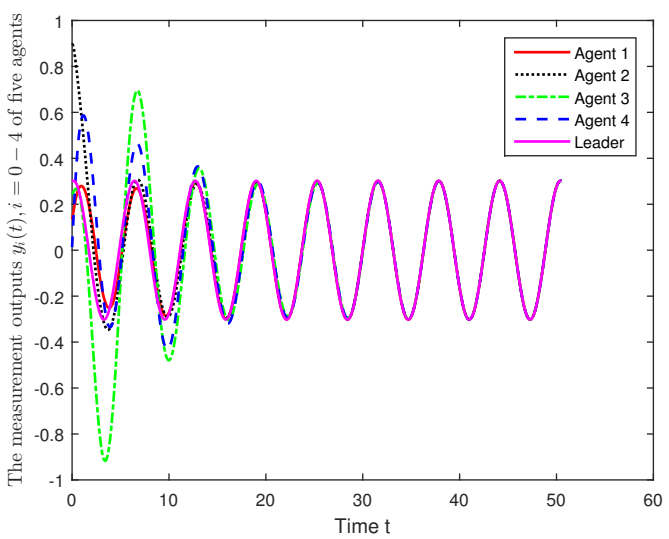

Figure 13. Example 2: the measurement outputs $y_{i}(t)$ of five agents

exosystem matrices $A_{0}$ and $C_{0}$ are given as $A_{0}=\left(\begin{array}{cc}0 & 1 \\ -1 & 0\end{array}\right)$ and $C_{0}=[1,0]$.

After simple calculation, the solutions of the regulator equations (11) can be obtained 
Cai, Y. L. et al.

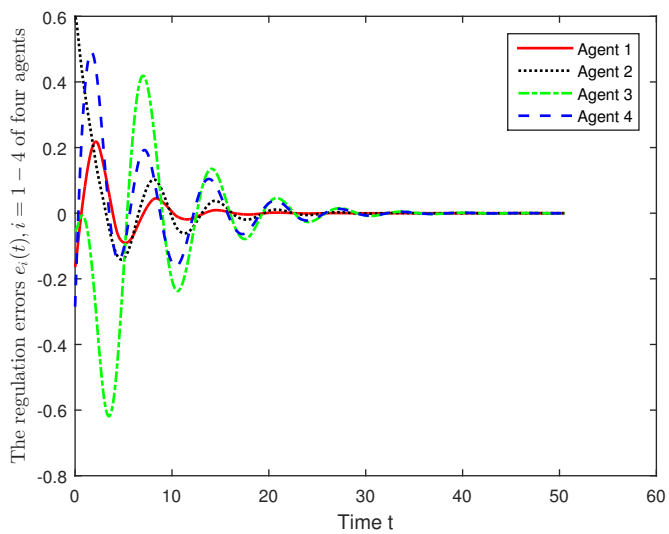

Figure 14. Example 2: the regulation errors $e_{i}(t)$ of four measurable agents

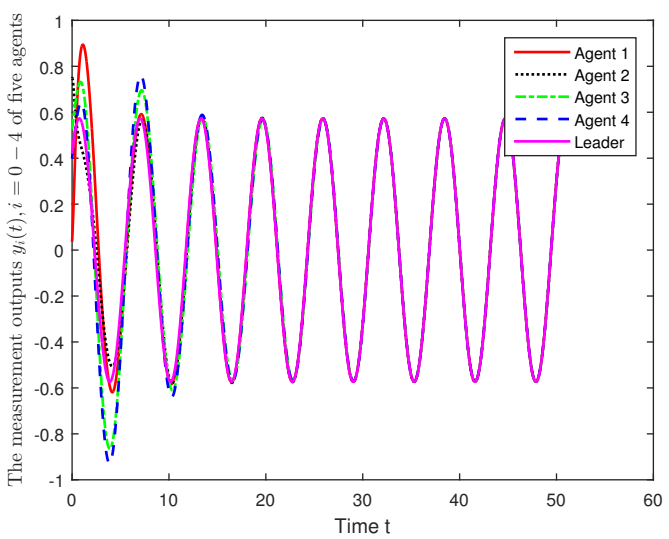

Figure 15. Example 2: the measurement outputs $y_{i}(t)$ of five agents

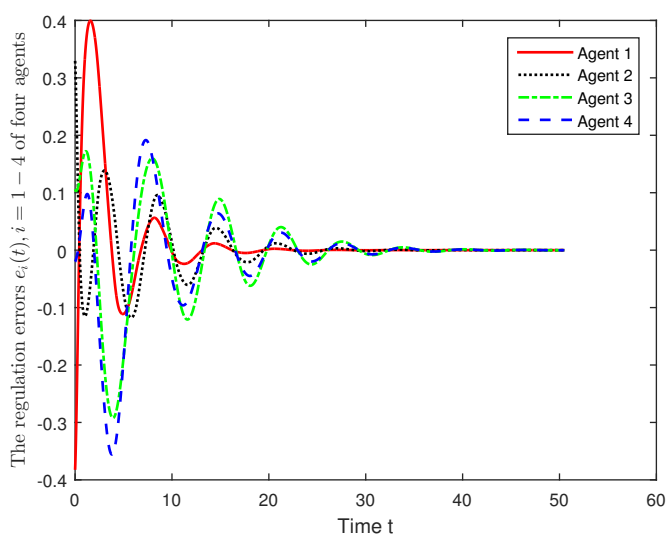

Figure 16. Example 2: the regulation errors $e_{i}(t)$ of four unmeasurable agents

$\Pi_{i}=\left(\begin{array}{ll}1 & 0 \\ 0 & 1\end{array}\right)(i=1,2, \ldots, 9), \quad \Gamma_{1}=[-2,0.5], \quad \Gamma_{2}=[1,-0.5], \quad \Gamma_{3}=[-1,0]$, $\Gamma_{4}=[0,-1], \Gamma_{5}=\Gamma_{6}=\Gamma_{7}=\Gamma_{8}=\Gamma_{9}=\Gamma_{4}$. The distributed control law (6) is designed with control gains $K_{11}=[-8,-4], K_{12}=[-5.5,1.5], K_{13}=[7,-4], K_{14}=[8,-4], K_{15}=$ $K_{16}=K_{17}=K_{18}=K_{19}=K_{14}, K_{21}=[6,4.5], K_{22}=[6.5,-2], K_{23}=[-8,4], K_{24}=$ $[-8,3], K_{25}=K_{26}=K_{27}=K_{28}=K_{29}=K_{24}$. Moreover, the regulation factor $\rho=-1$. The 
Cai, Y. L. et al.

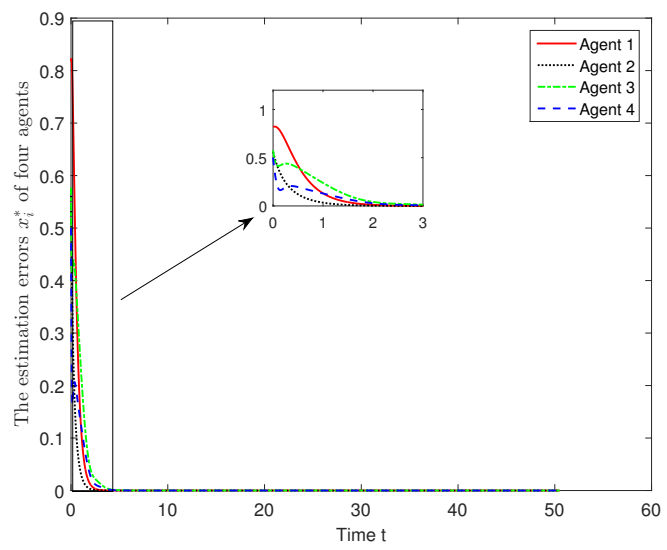

Figure 17. Example 2: the estimation errors $x_{i}^{*}$ of four unmeasurable agents

distributed control law (7) in [17] is designed with the same gain matrices.

The comparative experiment is implemented with the same initial states $x_{1}(0)=[2,-1]^{T}, x_{2}(0)=[-2,1]^{T}, x_{3}(0)=[0,-2]^{T}, x_{4}(0)=[0.5,-2]^{T}, x_{5}(0)=[3,1]^{T}$, $x_{6}(0)=[-1,-1]^{T}, x_{7}(0)=[3,1]^{T}, x_{8}(0)=[2,-1]^{T}, x_{9}(0)=[0.5,1.5]^{T}, \omega(0)=[0,0]^{T}$, and all other initial states are chosen to be zeros. The three-dimensional spatial output trajectories of [17] and our paper are presented in Fig. 18. And the regulation errors of [17] and our paper are given in Fig. 19. It can be seen that our method uses shorter time to track the external system. Hence, our controller is superior to [17] in terms of convergence speed. Moreover, the states of all agents are assumed to be measurable in [17]. However, in practice, it is difficult to measure all states due to high cost or technical constraint. For the case of unmeasurable states, the controller proposed in [17] is not applicable, while the controller proposed in this paper can solve this problem.

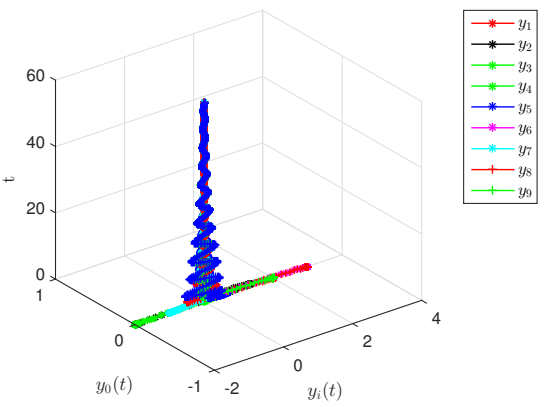

(a)

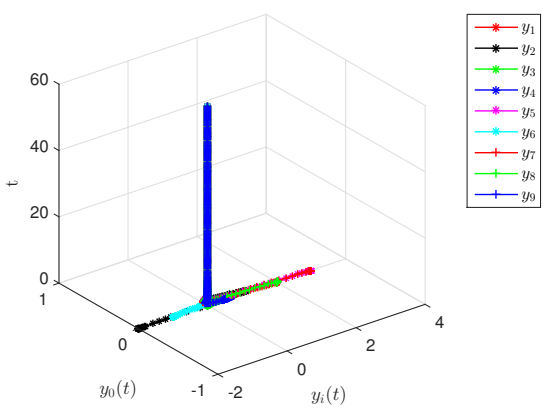

(b)

Figure 18. The 3D phase plane plot of all agents' output dynamics of (a) [17] and (b) this paper.

\subsection{Application examples}

In this section, to verify the practicability of the hybrid control protocol (6), the proposed controller is applied to the mobile robots and mass-damper-spring systems. The communication topology with seven mobile robots or seven mass-damper-spring systems is shown in Fig. 20. 
Cai, Y. L. et al.

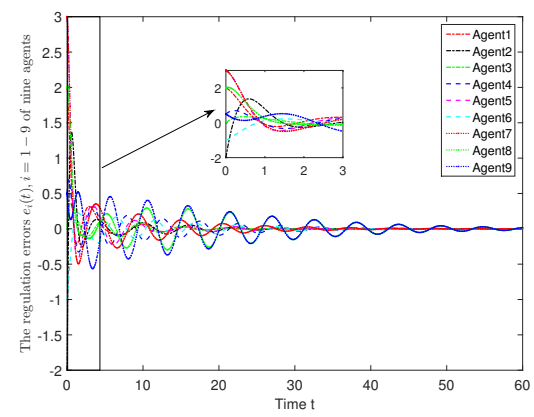

(a)

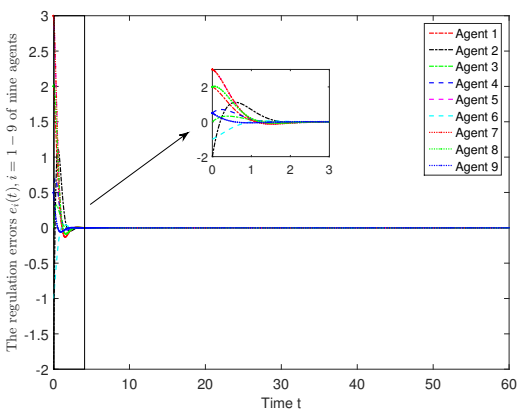

(b)

Figure 19. The regulation errors $e_{i}(t)$ of nine measurable agents of (a) [17] and (b) this paper.

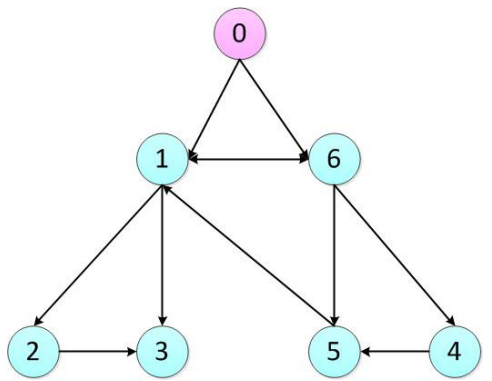

Figure 20. The communication topology among seven mobile robots or seven mass-damper-spring systems

In recent years, more and more research attention has been paid to the mobile robots due to its extensive applications such as universe exploration, factory automation and ocean development. Firstly, we consider the output regulation problem of seven mobile robots modeled as [32]. The system dynamics of each follower mobile robot are described as

$$
A_{i}=\left[\begin{array}{ccc}
0 & 1 & 0 \\
0 & 0 & c_{i} \\
0 & -d_{i} & -a_{i}
\end{array}\right], B_{i}=\left[\begin{array}{c}
0 \\
0 \\
b_{i}
\end{array}\right], E_{i}=\left[\begin{array}{cc}
0 & 0 \\
0 & 0 \\
0 & e_{i}
\end{array}\right], C_{i}=[1,0,0],
$$

where $a_{i}, b_{i}, c_{i}, d_{i}$ and $e_{i}$ are positive constants. The first two state components are considered as position and velocity and the third state component is regarded as an actuator state. The leader's dynamics are modeled by (2) with

$$
A_{0}=\left[\begin{array}{ll}
0 & 1 \\
0 & 0
\end{array}\right], C_{0}=[1,0] .
$$

The system parameters $f_{i}=\left[a_{i}, b_{i}, c_{i}, d_{i}, e_{i}\right], i=1, \ldots, 6$ are chosen as $f_{1}=[1,1,1,0,1], f_{2}=[10,2,1,0,1], f_{3}=[2,1,1,10,1], f_{4}=[2,1,1,1,3], f_{5}=[1,1,1,0,3]$, and $f_{6}=[10,2,1,0,3]$. The feedback gain matrices are selected as $K_{1}=[-27,-27,-8], K_{2}=[-13.5,-13.5,0.5], K_{3}=[-27,-17,-7], K_{4}=$ $[-27,-26,-7], K_{5}=[-27,-27,-8]$, and $K_{6}=[-13.5,-13.5,0.5]$. Moreover, by the Eq.

$(11)$

have

$$
\Pi_{i}=\left[\begin{array}{ll}
1 & 0 \\
0 & 1 \\
0 & 0
\end{array}\right], i=1, \ldots, 6,
$$

$\Gamma_{1}=[0,-1], \Gamma_{2}=[0,-0.5], \Gamma_{3}=[0,9], \Gamma_{4}=[0,-2], \Gamma_{5}=[0,-3]$, and $\Gamma_{6}=[0,-1.5]$. Furthermore, the regulation factor $\rho=-1$, and the distributed control law (7) in [17] is designed with the same gain matrices. 
Cai, Y. L. et al.

The comparative experiment is conducted with the same initial states as those in section 5.2. The regulation errors of [17] and our paper are shown in Fig. 21. It can be seen that the two controllers can be used for the output regulation of mobile robots, and our method uses shorter time to track the external system. Therefore, the hybrid feedback controller (6) has superior performance than [17] in terms of convergence speed.

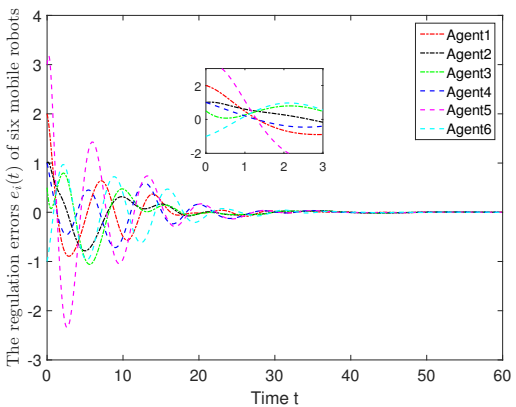

(a)

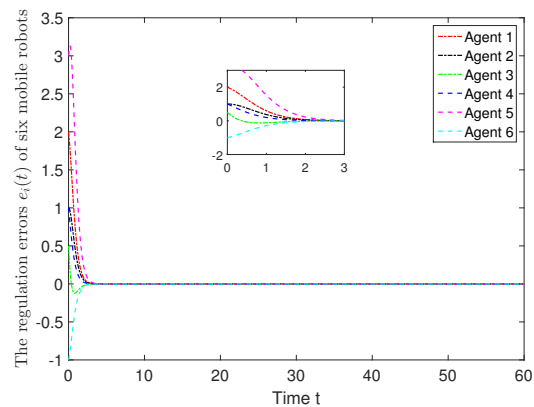

(b)

Figure 21. The regulation errors $e_{i}(t)$ of six mobile robots of (a) [17] and (b) this paper.

Secondly, we consider the output regulation problem of six mass-damper-spring systems and one harmonic oscillator [10]. The system dynamics of each mass-damper-spring system are given as

$$
\ddot{y}_{i}+g_{i} \dot{y}_{i}+h_{i} y_{i}=u_{i},
$$

where $u_{i}$ and $y_{i}$ are the input and output of $i$-th mass-damper-spring system, respectively. $g_{i}=0.6+0.2 i$ and $h_{i}=2.5-0.5 i$ are the damping coefficient and spring coefficient, respectively. Denoting $x_{i 1}=y_{i}$ and $x_{i 2}=\dot{y}_{i}$, the systems (37) can be expressed as

$$
\begin{aligned}
\dot{x}_{i} & =\left(\begin{array}{cc}
0 & 1 \\
-g_{i} & -h_{i}
\end{array}\right) x_{i}+\left(\begin{array}{l}
0 \\
1
\end{array}\right) u_{i}, \\
y_{i} & =(1,0) x_{i} .
\end{aligned}
$$

The harmonic oscillator is used for leader, and its dynamics are as follows

$$
\begin{aligned}
& \dot{x}_{0}=\left(\begin{array}{cc}
0 & \varpi \\
-\varpi & 0
\end{array}\right) x_{0}, \\
& y_{0}=(1,0) x_{0},
\end{aligned}
$$

where $\varpi=1$.

The feedback gain matrices are selected as $K_{1}=[-27,-27], K_{2}=[-13.5,-13.5], K_{3}=$ $[-27,-17], K_{4}=[-27,-26], K_{5}=[-27,-27]$, and $K_{6}=[-13.5,-13.5]$. Moreover, by the Eq. (11), we can obtain $\Pi_{i}=\left[\begin{array}{ll}1 & 0 \\ 0 & 1\end{array}\right], i=1, \ldots, 6, \Gamma_{1}=[2.0,0.8], \Gamma_{2}=[1.5,1.0], \Gamma_{3}=$ $[1.0,1.2], \Gamma_{4}=[0.5,1.4], \Gamma_{5}=[0,1.6]$, and $\Gamma_{6}=[-0.5,1.8]$. Furthermore, the regulation factor is selected as $\rho=-1$ and the distributed control strategy (7) in [17] is designed with the same gain matrices.

The comparative experiment is also conducted with the same initial states as those in section 5.2. The simulation results are presented in Fig. 22. One can get that the two control strategies can be used for the output regulation of mass-damper-spring systems, and our method takes shorter time to track the external system. Hence, our control strategy is superior to [17] in terms of convergence speed. 
Cai, Y. L. et al.

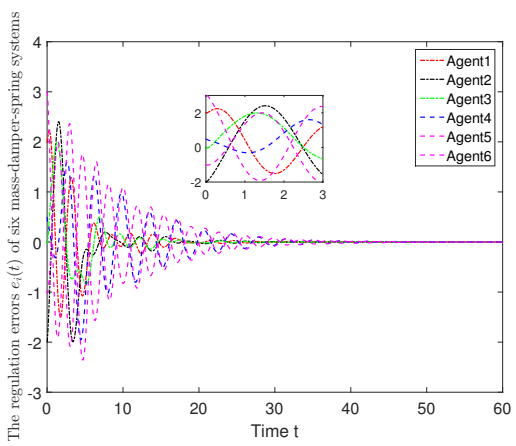

(a)

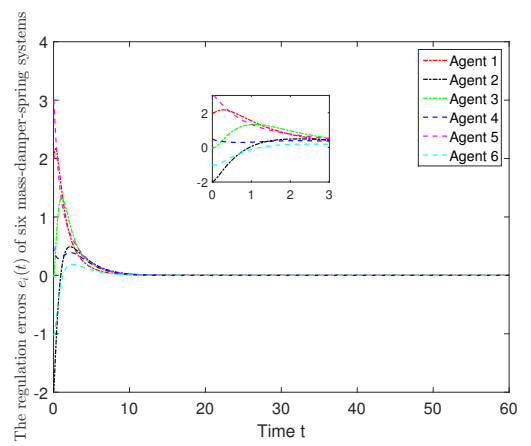

(b)

Figure 22. The regulation errors $e_{i}(t)$ of six mass-damper-spring systems of (a) [17] and (b) this paper.

\section{Conclusion}

In this work, we have considered the output regulation problem of linear heterogeneous MASs with partial unmeasurable states under switching topology. The agents excluding the external system have been divided into two groups with measurable agents or unmeasurable agents. For the unmeasurable agents, we have constructed the full-order Luenberger observer. Moreover, a dynamic compensator has been designed for these agents that cannot obtain the information from the external system directly. Then a hybrid control strategy with the designed observer and compensator has been proposed to address the output regulation issue. Simulation results have denoted that the proposed controller is feasible and promising for the output regulation issue of linear heterogeneous MASs under switching topology. Future research along this direction will address the output regulation issue of nonlinear MASs with time delay under switching topology. How to choose the nodes to obtain influence maximization is also the future research direction [33, 34].

\section{Acknowledgments}

We are very grateful to the editors and reviewers for their suggestions on our paper. This work was supported by the Fundamental Research Funds for the Central Universities under Grant No. N171706002.

\section{Conflicts of Interest}

There is no conflict of interest.

\section{References}

[1] Zeng, X., Liu, Z., and Hui, Q. (2015). Energy equipartition stabilization and cascading resilience optimization for geospatially distributed cyber-physical network systems. IEEE Transactions on Systems, Man, and Cybernetics: Systems, 45(1), 25-43.

[2] He, W., Ge, W., Li, Y., Liu, Y. J., Yang, C., and Sun, C. (2016). Model identification and control design for a humanoid robot. IEEE Transactions on Systems, Man, and Cybernetics: Systems, 47(1), 45-57.

[3] Cai, Y., Zhang, H., Zhang, K., and Liang, Y. (2019). Distributed leader-following consensus of heterogeneous second-order time-varying nonlinear multi-agent systems under directed switching topology. Neurocomputing, 325, 31-47. 
[4] Wang, B., Wang, J., Zhang, B., Chen, W., and Zhang, Z. (2018). Leaderfollower consensus of multivehicle wirelessly networked uncertain systems subject to nonlinear dynamics and actuator fault. IEEE Transactions on Automation Science and Engineering, 15(2), 492-505.

[5] Cai, Y., Zhang, H., Zhang, K., and Liu, C. (2019). Fuzzy adaptive dynamic programming-based optimal leader-following consensus for heterogeneous nonlinear multi-agent systems. Neural Computing and Applications, DOI: https://doi.org/10.1007/s00521-019-04263-0.

[6] Liang, H., Zhang, Y., Huang, T., and Ma, H. (2019) Prescribed performance cooperative control for multiagent systems with input quantization. IEEE transactions on cybernetics, DOI: 10.1109/TCYB.2019.2893645.

[7] Yaghmaie, F. A., Lewis, F. L., and Su, R. (2016). Output regulation of linear heterogeneous multi-agent systems via output and state feedback. Automatica, 67, $157-164$.

[8] Liang, H., Zhang, Z., and Ahn, C.K. (2019) Event-triggered fault detection and isolation of discrete-time systems based on geometric technique. IEEE Transactions on Circuits and Systems II: Express Briefs, DOI: 10.1109/TC-SII.2019.2907706.

[9] $\mathrm{Hu}, \mathrm{W}$., and Liu, L. (2017). Cooperative output regulation of heterogeneous linear multi-agent systems by event-triggered control. IEEE transactions on cybernetics, 47(1), 105-116.

[10] Chu, H., Gao, L., and Zhang, W. (2016). Distributed adaptive containment control of heterogeneous linear multi-agent systems: an output regulation approach. IET Control Theory and Applications, 10(1), 95-102.

[11] Hu, W., Liu, L., and Feng, G. (2018). Robust cooperative output regulation of heterogeneous uncertain linear multi-agent systems by intermittent communication. Journal of the Franklin Institute, 355(3), 1452-1469.

[12] Wu, Y., and Hu, J. (2018). Observer-based output regulation of cooperative-competitive high-order multi-agent systems. Journal of the Franklin Institute, 355(10), 4111-4130.

[13] Yan, F., Gu, G., and Chen, X. (2018). A new approach to cooperative output regulation for heterogeneous multi-agent systems. SIAM Journal on Control and Optimization, 56(3), 2074-2094.

[14] Xiang, J., Li, Y., and Hill, D. J. (2017). Cooperative output regulation of linear multiagent network systems with dynamic edges. Automatica, 77, 1-13.

[15] Cai, H., Lewis, F. L., Hu, G., and Huang, J. (2017). The adaptive distributed observer approach to the cooperative output regulation of linear multi-agent systems. Automatica, 75, 299-305.

[16] Yang, R., Zhang, H., Feng, G., Yan, H., and Wang, Z. (2019) Robust cooperative output regulation of multi-agent systems via adaptive event-triggered control. Automatica, $102,129-136$.

[17] Lu, M., and Liu, L. (2017) Cooperative output regulation of linear multi-agent systems by a novel distributed dynamic compensator. IEEE Transactions on Automatic Control, 62(12), 6481-6488.

[18] Li, X., Soh, Y. C., Xie, L., and Lewis, F. L. (2018). Cooperative output regulation of heterogeneous linear multi-agent networks via $H_{\infty}$ performance allocation. IEEE Transactions on Automatic Control, 64(2), 683-696. 
[19] Schmid, R., and Aghbolagh, H. D. (2018). Nonovershooting cooperative output regulation of linear multi-agent systems by dynamic output feedback. IEEE Transactions on Control of Network Systems, DOI: 10.1109/TCNS.2018.2846180.

[20] Abdessameud, A., and Tayebi, A. (2018) Distributed output regulation of heterogeneous linear multi-agent systems with communication constraints. Automatica, $91,152-158$.

[21] Huang, J. (2017) The cooperative output regulation problem of discrete-time linear multi-agent systems by the adaptive distributed observer. IEEE Transactions on Automatic Control, 62(4), 1979-1984.

[22] Qian, Y.Y., Liu, L., and Feng, G. (2019) Distributed dynamic event-triggered control for cooperative output regulation of linear multiagent systems. IEEE transactions on cybernetics, DOI: 10.1109/TCYB.2019.2905931.

[23] Zhang, X., and Lewis, F. L. (2018). Cooperative output regulation of heterogeneous multi-agent systems based on passivity. International Journal of Systems Science, 49(16), 3418-3430.

[24] Li, S., Feng, G., Wang, J., Luo, X. and Guan, X. (2015) Adaptive control for cooperative linear output regulation of heterogeneous multi-agent systems with periodic switching topology. IET Control Theory and Applications, 9(1), 34-41.

[25] Hu, W., Liu, L., and Feng, G. (2019) Event-triggered cooperative output regulation of linear multi-agent systems under jointly connected topologies. IEEE Transactions on Automatic Control, 64(3), 1317-1322.

[26] Wang, C., Pan, H., Zheng, M., and Gao, J. (2018) Cooperative robust output regulation of linear multi-agent systems under switching networks. In 2018 Ninth International Conference on Intelligent Control and Information Processing (ICICIP), 295-300.

[27] Yan, Y., and Huang, J. (2017) Cooperative output regulation of discrete-time linear time-delay multi-agent systems under switching network. Neurocomputing, 241, 108114.

[28] Godsil, C. and Royle, G.F. (2013) Algebraic graph theory. Springer Science \& Business Media.

[29] Cheng, B., Wang, X., and Li, Z. (2018) Event-triggered consensus of homogeneous and heterogeneous multiagent systems with jointly connected switching topologies. IEEE transactions on cybernetics, (99), 1-10.

[30] Wu, Z.G., Xu, Y., Lu, R., Wu, Y. and Huang, T. (2017) Event-triggered control for consensus of multiagent systems with fixed/switching topologies. IEEE Transactions on Systems, Man, and Cybernetics: Systems, (99), 1-11.

[31] Liang, H., Zhang, H., Wang, Z., and Feng, T. (2016) Reduced-order observer-based distributed tracking control for high-order multi-agent systems with heterogeneous leader. Journal of the Franklin Institute, 353(11), 2511-2533.

[32] Zhang, D., Xu, Z., Karimi, H. R., Wang, Q. G., and Yu, L. (2018). Distributed $H_{\infty}$ output-feedback control for consensus of heterogeneous linear multiagent systems with aperiodic sampled-data communications. IEEE Transactions on Industrial Electronics, 65(5), 4145-4155.

[33] He, Q., Wang, X., Lei, Z., Huang, M., Cai, Y., and Ma, L. (2019). TIFIM: A Twostage Iterative Framework for Influence Maximization in Social Networks. Applied Mathematics and Computation, 354, 338-352. 
Cai, Y. L. et al.

[34] He, Q., Wang, X., Huang, M., Lv, J., and Ma, L. (2018). Heuristics-based influence maximization for opinion formation in social networks. Applied Soft Computing, 66, 360-369. 